\title{
Über die Einwirkung des Zuckers auf die Verdauung.
}

\author{
Von \\ Erwin Thomsen. \\ Mit einer Tafel. \\ (Der Redaktion zugegangen am 23. März 1913.)
}

Die Wirkung des Zuckers auf die Verdauung ist bisher kaum untersucht worden. Clemm, ${ }^{1}$ ) der am Kleinen Magen Versuche gemacht hat, gibt an, daß die Magensaftsekretion durch Zucker verringert würde. Best und Cohnheim²) sahen, da $B$ bei Durchlaufversuchen am leeren Magen Zuckerlösungen den Magen langsamer verließen als Salzlösungen. Best ${ }^{3}$ ) beschreibt einen Versuch mit festem Zucker an einem Hunde mit Duodenalfistel. Über die Einwirkung des Zuckers, der zu anderer Nahrung hinzugegeben wird, fehlen alle Angaben.

$\mathrm{DaB}$ aber der Zucker, der zu anderen Speisen hinzukommt, einen Einfluß auf die Verdauung hat, ist nach Beobachtungen am Menschen zu erwarten. Viele Menschen können nüchtern süße Speisen nicht vertragen. Nach Genuß von Zucker oder Süßigkeiten auf den leeren Magen bekommen sie Sodbrennen und verlieren auf eine gewisse Zeit den Appetit. Anderseits hat man bei Patienten von der Zufuhr größerer Zuckermengen auch günstige Erfolge gesehen. Endlich ist es schon seit langer Zeit bei den meisten Kulturvölkern Sitte, am Schlusse der Hauptmahlzeit eine Torte, Pudding oder sonstige Süßspeisen zu genießen. Es war wohl denkbar, daß auch

1) W. N. Clemm, Therap. Monatshefte, 1901, S. 402.

2) F. Best und O. Cohnheim, Diese Zeitschrift, Bd. 69, S. 117, 1910.

3) F. Best, Deutsch. Arch. f. klin. Med., Bd. 104, S. 94, 1911. 
dies, wie viele andere Gewohnheiten des Menschen, einen physiologischen Grund hat.

Ich habe daher auf Veranlassung von Herrn Professor Cohnheim in seinem Laboratorium Versuche über die Verdauung von Zucker und die Einwirkung von Zucker auf die Verdauungsorgane an Hunden mit seitenständigen Darmfisteln gemacht.

DieVersuchsanordnung ist wiederholt beschrieben worden. ${ }^{1}$ ) Die Hunde wurden bei offener Duodenalfistel gefüttert. Was ins Duodenum kommt, entleert sich dabei aus der Kanüle, das Entleerte wird aufgefangen, durch ein Drahtnetz filtriert und aus einer graduierten Bürette möglichst schnell durch die Einspritzvorrichtung abwärts von der Kanüle in den Darm einlaufen gelassen. Bei diesem Verfahren kann man natürlich Magenoder Darminhalt nicht untersuchen oder höchstens einzelne Proben entnehmen. Aber man hat die natürlichen Verhältnisse denkbar vollkommen nachgeahmt. Während des ganzen Verlaufes wird von jedem Quantum, das herauskommt, Zeit und Menge notiert. So erhält man die Gesamtentleerungsdauer, die Gesamtmenge der Sekrete, außerdem aber auch den Verlauf der Entleerung des Magens und der Sekretionen, der gerade bei meinen Versuchen ein erhebliches Interesse gewann. Es ist auch möglich, und ich habe es getan, diesen Verlauf eines Versuches graphisch aufzuzeichnen.

$\mathrm{Zu}$ den Versuchen standen mir 4 Hunde zur Verfügung. Hund Pascha, der eine Magen- und Duodenalfistel hatte. Hund Wastl, der eine Fistel im Duodenum und eine zweite im untersten Ileum hatte.

Hund Nero, der eine Duodenalfistel hatte und bei dem außerdem die Galle aus dem Duodenum abgeleitet war. Es ist derselbe Hund, der seinerzeit von Cohnheim und Klee ${ }^{2}$ ) beschrieben worden ist.

1) 0. Cohnheim, Abderhaldens Handb. d. biochem. Arbeitsmethoden, Bd. 6, S. 564, 1912. - L. Klocmann, Diese Zeitschrift, Bd. 80, S. 17, 1912.

2) O. Cohnheim und P. Klee, Diese Zeitschrift, Bd. 78, S. 464, 1912. 
Hund Caesar, der eine Fistel im Duodenum und eine solche im Coecum hatte.

Zuerst galt es, bei den Versuchen festzustellen, ob durch Hinzufügen von Zucker die normale Verweildauer der Nahrung im Magen verändert würde, oder ob sich eventuell eine veränderte Sekretabsonderung zeigte. Die ersten 4 Versuche, die bei Hunden mit Duodenalfisteln gemacht wurden, denen $150 \mathrm{~g}$ Fleisch teils mit Zugabe von $25 \mathrm{~g}$ Streuzucker, teils ohne Zucker gegeben wurden, schienen keine wesentlichen Vergrößerungen der Sekretmengen, auch keine Verlängerung der gesamten Zeitdauer des Versuches zu erbringen.

Versuch 1. Pascha: $150 \mathrm{~g}$ Fleisch $+25 \mathrm{~g}$ Streuzucker. Nach 240 Min.: $353 \mathrm{ccm}$ flüssige Bestandteile +177 g Festes, $355 \mathrm{ccm}$ Sekret.

Versuch 2. - : $150 \mathrm{~g}$ Fleisch. Nach 231 Min.: $419 \mathrm{ccm}$ Flüssiges $+138 \mathrm{~g}$ Festes, $407 \mathrm{ccm}$ Sekret.

Versuch 3. Nero : $125 \mathrm{~g}$ Fleisch $+40 \mathrm{~g}$ Streuzucker. Nach 187 Min.: $167 \mathrm{ccm}$ Flüssiges $+94 \mathrm{~g}$ Festes.

Versuch 4. - : $125 \mathrm{~g}$ Fleisch. Nach 189 Min.: $152 \mathrm{ccm}$ Flüssiges $+89 \mathrm{~g}$ Festes.

Die letzteren beiden Versuche erachte ich aber noch nicht für beendet, und es fiel mir bei diesen Versuchen auf, daß bei Zuckerzugabe in der Reihenfolge der Entleerung Unregelmäßigkeiten eintraten. Bei den Normalversuchen über die Verdauung im Magen fanden Cohnheim und Dreyfus, daß die Speisen in regelmäßigen Zeiträumen den Magen verlassen. Während der ganzen Zeit der Verdauung tritt in den Zeiträumen keine wesentliche Veränderung ein. Ganz anders wird es nun; wenn der Nahrung Zucker entweder hinzugefügt oder gleich hinterher eine Zuckermahlzeit verabreicht wird. Besonders war es der Versuch 1, der für die Zuckerversuche als charakteristisches Beispiel gelten kann. Das Protokoll gibt Kubikzentimeter Flüssigkeit. Das Feste wurde von Zeit zu Zeit gewogen. Ich habe zur besseren Übersicht diesen Versuch auf Millimeterpapier darzustellen versucht. Fig. 1 zeigt diesen Versuch an. Abszisse Zeit, Ordinaten Kubikzentimeter. 
Erster Schuß 7 Min.

10 Min. $10 \mathrm{ccm}$

$16 \quad 12$

$20 \quad 8$ Galle

$24 \quad 8$

$27 \quad 10$

$30 \quad 21 / 2$

$36 \quad 8$ Fleisch m. Schleim

$38 \quad 11$

429

$49 \quad 3 \frac{1}{2}$ Fleisch

$54 \quad 31 / 2$,

614

$68 \quad 4$ mit Schleim durchsetztes Fleisch

\section{6}

78

81

83

87

90

96

99

101

106

110

116

120

(23 g Festes)

(35 g Festes)

2

2

3

$21 / 2$ Galle

(40 g Festes)

10

8

10

Galle ohne Fleisch

Fleisch 2
122 Min. $8 \mathrm{ccm}$

1326

13510 kein Fleisch

$141 \quad 11$ Fleisch

1446

$148 \quad 5$

$151 \quad$ (39 g Festes)

152 ว

1557

$157 \quad 4$

$160 \quad 7$

16399

$167 \quad 4$

$171 \quad 4$

$174 \quad 5$

$177 \quad 6$

$181 \quad 12$

$191 \quad 14$

$193 \quad 10$

$199 \quad 8$

$205 \quad 7$

$209 \quad 22$

2217

$227 \quad 5$

$235 \quad 27$

240 Magen geöffnet und ausgespült.

Noch 40 g Fleisch.

Hier ist das Hauptaugenmerk auf die Zeit zwischen 30 und 90 Minuten nach der Fütterung zu richten. Bei 30 hört plötzlich jede Sekretion auf, statt dessen aber wird in großen Schüssen Fleisch entleert, ohne jegliche Flüssigkeit, während 30 Minuten hindurch. Dann lassen die Fleischschüsse nach und mischen sich mit flüssigem Sekret. Ungefähr 20 Minuten später kommt noch einmal ein starker Fleischschuß, dem nun die flüssige Sekretion in der bisherigen Höhe folgt.

Bei den darauf folgenden Probefrühstücksversuchen mit Zucker trat ebenfalls eine Konstitutionsveränderung ein, die sich dadurch bemerkbar machte, daß der zu erwartende saure Brotbrei zeitweilig ausblieb, und in der Pause Pankreassaft . . und Galle kamen, sodaß wir schon häufig glaubten, der Magen 
sei leer oder er hätte doch seine Funktion eingestellt. Das Probefrühstück bestand bei diesen Versuchen aus $400 \mathrm{ccm}$ Wasser +50 g Brot und 25 g Zucker. Als Beispiel gebe ich:

\section{Versuch 5. P'ascha.}

Bis zu 20 Minuten war die Sekretion wie bei jedem gewöhnlichen Probefrühstück, dann traten plötzlich starke Schüsse auf, die, rasch aufeinanderfolgend, eine große Menge Brot entleerten. (Bei 24 kamen sogar kurz hintereinander $15+11$ $+7 \mathrm{ccm}$ heraus.) Dann verlangsamte sich die Sekretion, bis bei 32 Minuten nur Tropfen herauskamen und kein Brot mehr. Die Reaktion wurde nun alkalisch. Der eingetretene Stillstand hielt bis 42 an. Leider konnte der Versuch nicht fortgesetzt werden, da infolge des Stillstandes Rücklauf eingetreten war.

Nach 9 Min. erster Schuß

$9 \mathrm{Min} .16 \mathrm{ccm}$

109

12

15

18

20

21

22

24

26

27

28

29

30

31

32

33

35

40
Galle

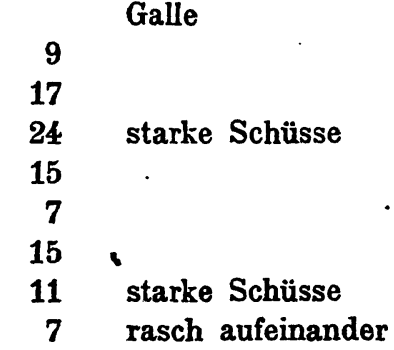

folgend

12

9

18

15

reichlich Galle

5

6

9

7 Tropfen, keine

Schüsse

\section{3}

2 alkalisch

2 Galle

Stillstand
42 Min. saurer Schuß

45

$5 \mathrm{ccm}$

Kongo -

$\begin{array}{lll}46 & 7 & \text { regelmäß. Schüsse }\end{array}$

$47 \quad 11$

487 Kongo + st.

49

schwach sauer

Kongo -, Galle

st. sauer Kongo +

st. sauer Kongo +

514

$52 \quad 8$

$53 \quad 10 \quad$.. starke Schüsse

$54 \quad 15$

$56 \quad 22$

Rücklauf ?

58

59

61

63

67

72

73

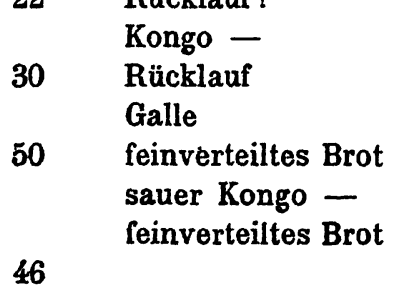




\section{Versuch 6. Nero:}

Hier verlief der Anfang wie bei Versuch 5. Bei 16 Min. traten die ersten starken Schüsse auf, die dann bei 21' langsamer wurden, bis bei $32^{\prime}$ ein kurzer Stillstand eintrat. Bei $36^{\prime}$ begann wieder saure Reaktion, der dann bei $40^{\prime}$ wieder Schüsse folgten. Dies blieb so bei bis $66^{\prime}$, dann trat wieder Stillstand ein, der sich bis $83^{\prime}$ hinzog.

\begin{tabular}{|c|c|c|c|c|c|c|c|}
\hline \multicolumn{2}{|c|}{9 Min. erster Schuß } & \multicolumn{3}{|c|}{$40 \mathrm{Min} .6 \mathrm{ccm}$} & \multicolumn{3}{|c|}{$122 \mathrm{Min} .3 \mathrm{ccm}$} \\
\hline 12 & $.10 \mathrm{ccm}$ & 41 & 4 & & 131 & & Kongo + \\
\hline 14 & 19 & 43 & 8 & sauer & 133 & 6 & \\
\hline 15 & 29 & & & Kongo - & 135 & 6 & \\
\hline 16 & 28 & 46 & 7 & Kongo + & 137 & 8 & \\
\hline 17 & 22 & 48 & 8 & & 139 & 5 & \\
\hline 18 & 15 & 49 & 31 & & 141 & 3 & \\
\hline 19 & 20 & 59 & 8 & & 149 & 2 & \\
\hline 20 & 11 & 61 & 13 & Brot & 157 & & Brot \\
\hline 21 & 9 & 66 & 8 & viel Brot & 167 & 15 & stark \\
\hline 24 & 8 & 70. & 10 & Pause & & & sauer \\
\hline 25 & 6 & 83 & 6 & Brot & 184 & 3 & \\
\hline 28 & 5 & 88 & 7 & Brot & 196 & 3 & Kongo + \\
\hline 31 & 9 & 95 & 5 & & 200 & 8 & \\
\hline 32 & 7 & 103 & 4 & Alkali & 216 & 13 & Kongo + \\
\hline 33 & j & 108 & 2 & kein Brot & 219 & 7 & \\
\hline 34 & 5 sauer & 110 & & Brot & 225 & 4 & \\
\hline 36 & 8 sauer & 112 & 6 & & 236 & 4 & \\
\hline & Kongo - & 116 & 6 & & 239 & & \\
\hline 39 & 9 & 119 & 6 & & & & \\
\hline
\end{tabular}

Sodann machte ich einen Versuch mit einer Probemahlzeit, der ich in Form einer Nachspeise einen kleinen Zuckerkuchen nachfolgen ließ.

\section{Versuch 7. Wastl.}

$250 \mathrm{ccm}$ Bouillon aus einem Bouillonwürfel. $150 \mathrm{~g}$ Fleisch als Beefsteak gebraten mit 20 g Butter. 300 g Kartoffelpüree. Fleisch und Kartoffeln wurden gut durcheinander gemengt. 10 Minuten nach dem Fressen bekam der Hund als Nachspeise $25 \mathrm{~g}$ Albert-Cakes, fein gepulvert mit $40 . \mathrm{g}$ Streuzucker und $40 \mathrm{ccm}$ Milch zu einem Kuchen verrieben. 
Über die Einwirkung des Zuckers auf die Verdauung.

431

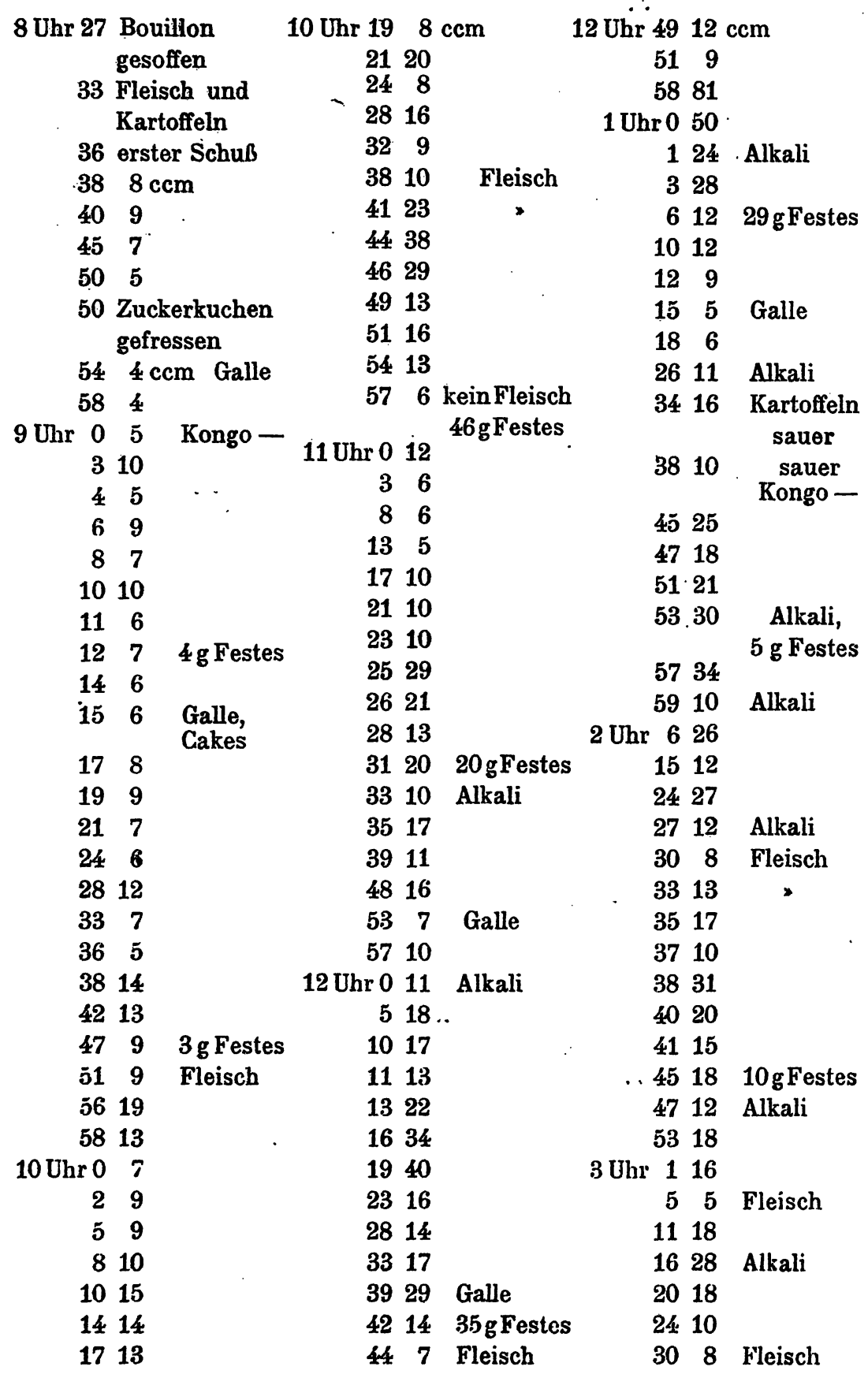




\begin{tabular}{|c|c|c|c|}
\hline \multicolumn{2}{|c|}{3 Uhr 36 25 ccm } & 4 Uhr $915 \mathrm{ccm}$ & \multirow{2}{*}{$\begin{array}{l}\text { Tier säuft, Magen leer. } \\
1923 \mathrm{ccm} \text { Flüssigkeit }\end{array}$} \\
\hline 1121 & Alkali & 1211 Alkali & \\
\hline 129 & $13 \mathrm{~g}$ Festes & 1510 & 165 g Festes \\
\hline 1517 & & 163 & 1288 , Sekret \\
\hline 5112 & & 21 & (normale Probemahlzeit \\
\hline $\begin{array}{ll}59 & 9\end{array}$ & Fleisch & 25 & $1200)$ \\
\hline \& Uhr \& 19 & & 30 & \\
\hline
\end{tabular}

Das Resultat des Versuches ist auch graphisch dargestellt in Fig. 2. Hier stellen die punktierten Linien die Zeiten und Mengen dar, bei welchen nur reiner Pankreassaft, vermischt mit Galle, den Magen verlassen hat. Die ausgezogenen Linien geben an, zu welchen Zeiten und mit wie großen Schüssen sich Mageninhalt entleerte. Dieser stundenlange Versuch zeigt, wie Galle und Pankreassaft mit Fleischausscheidung regelmäßig abwechseln. Durchschnittlich beträgt die Zeit jeder einzelnen Ausscheidung ca. 30 Minuten. Die Pankreas-Gallensekretion bleibt durchschnittlich auf demselben Niveau. Die Fleischentleerung hingegen macht große Sprünge. Meistens beginnen diese Fleischausstoßungen mit einem starken Schuß, dem nach einiger Zeit ein zweiter, nicht ganz so großer folgt. Dann tritt eine Verlangsamung ein, die dann übergeht in die Pankreassekretion. Bei diesem Versuch ist besonders eigentümlich.der große. Schuß von $74 \mathrm{ccm}$, der um $1 \mathrm{Uhr}$ von dem Hunde ausgeschieden wurde. Das Übrige ist wohl am besten aus der Kurve selbst zu lesen, der (Fig. 3) eine Kurve beigefügt ist, die einen Normalversuch darstellt, bei dem nicht so hohe Sprünge vorkommen, auch die Fleischausscheidung regelmäßig bleibt, bis zum Schluß.

Um zu kontrollieren, ob diese Eigentümlichkeiten von dem Magen allein ausgingen, wurde bei dem Hunde Wastl, der eine Duodenal- und eine tiefe Ileumfistel hat, noch ein Versuch gemacht.

\section{Versuch 8. Wastl.}

Der Hund erhielt ein Frühstück, bestehend aus $50 \mathrm{~g}$ Brot und $50 \mathrm{ccm}$ Wasser. Der Versuch wurde 15 Minuten lang normal fortgeführt. Dann wurde dem aus der Duodenalkanüle Aufgefangenen 2-10 ccm einer 50\%igen Zuckerlösung $\mathrm{zu}-$ 
gefügt und wieder eingespritzt. Dabei stellte es sich heraus, daß nach etwa 30 Minuten nach der ersten Einspritzung des Zuckers das Brot plötzlich wegblieb, und nur Galle und Pankreassaft ausgeschieden wurde. Die Ausscheidung des Brotes begann erst wieder nach 55 Minuten.

Der Versuch beweist, daß die Verzögerung der Magenentleerung und das Auftreten von Pausen, in denen sich Pankreassaft und Galle entleeren, keine Magenwirkung darstellt, sondern durch eine Einwirkung des Zuckers auf den Dünndarm zustandekommt. Dieselbe Erscheinung, daß Arzneimittel nicht primär auf den Magen wirken, sondern reflektorisch vom Dünndarme aus, Sekretion und Motilität des Magens beeinflussen, ist von

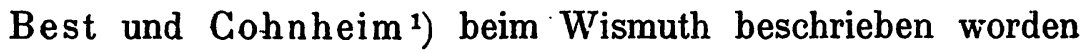
und ist vermutlich auch bei den Stoffen der Fall, deren Einwirkung auf den Magen Klocmann beobachtet hat. ${ }^{2}$ )

Wie wirkt nun der Zucker auf den Dünndarm? Um dies herauszubringen, habe ich den Hunden in nüchternem Zustande Zuckerlösungen in die Duodenalfistel einlaufen lassen. Es ergab sich, daß Zuckerlösungen von 5 und 10\% auf Pankreassaft und Galle nicht anders wirken als reines Wasser. Auf diese Sekrete hat Zucker also keine spezifische Wirkung. Sodann ließ ich dem Hund Wastl mit Duodenal- und Ileumfistel $500 \mathrm{ccm}$ einer' 10\% igen Rohrzuckerlösung in die Duodenalkanüle einlaufen. Schon nach wenigen Minuten begann sich die Zuckerlösung in großen Schüssen aus der Ileumfistel zu entleeren. Bis auf $80 \mathrm{ccm}$ wurde die gesamte Menge wiedergefunden. Die aufgefangene Lösung gab eine nur eben angedeutete $\mathrm{Feh}$ lingsche Reaktion. Nach Kochen mit verdünnter Salzsäure wurde der Zuckergehalt auf Invertzucker berechnet, nach Bertrand bestimmt. Die Lösung enthielt 9,7\% Zucker. Es war also relativ wenig resorbiert worden und eine Spaltung kaum eingetreten. Die Zuckerlösung verhielt sich also nicht anders, als es Kochsalzlösungen nach $\mathrm{Best}^{3}$ ) tun, die auch rasch hindurchlaufen und von denen nur wenig resorbiert wird.

1) F. Best u. O. Cohnheim, Münch. med. Wochenschr., 1911, Nr. 51.

2) Klocmann, Diese Zeitschrift, Bd. 80, S. 17, 1912.

3) F. Best, Rostocker Habilitationsschr., 1912. 
Durch eine einfache Einwirkung des Zuckers kann also die eigentümliche Magenwirkung nicht erklärt werden. Ich habe daher die Hunde Wastl und Caesar Zuckerlösungen saufen lassen. Wenn sie es nicht freiwillig taten, wurde eine kleine Menge Milch hinzugetan, die sich im Laboratorium als bestes Geschmackkorrigens für Hunde bewährt hat. Nach Best besteht bei Salzlösungen beim nüchternen, nicht durstigen Hunde kaum ein Unterschied, ob man die Hunde saufen läßt, oder ob man die Lösung mit Umgehung des Magens direkt in den Dünndarm einführt. Ganz anders bei Zucker.

Hund Wastl: Der Hund soff $970 \mathrm{ccm}$ einer 10\%igen Zuckerlösung. In $11 / 2$ Stunden wurden aus der lleumfistel nur $13 \mathrm{ccm}$ aufgefangen. Diese zeigten bei der Untersuchung auf Zucker nach Bertrand 0,6\% Zucker und nach Spaltung des noch vorhandenen Rohrzuckers mittels Salzsäure noch 3,6\%. Die Duodenalfistel blieb bei diesem Versuch geschlossen.

Hund Caesar: Der Hund säuft 21 einer 10\% igen Zuckerlösung. Duodenalfistel geschlossen. Nach 2 Stunden konnte man aus der Coecumfistel $14 \mathrm{ccm}$ auffangen, die nach Bertrand $0,26 \%=0,4 \mathrm{~g}$ enthielten und nach Spaltung des Zuckers $0,57 \%=0,8 \mathrm{~g}$.

Hund Caesar: Dàs Tier frißt $100 \mathrm{~g}$ Zucker in Substanz. Duodenalfistel geschlossen. In 2 Stunden entleeren sich aus dem Coecum $50 . \mathrm{ccm}$ von etwas dünnbreiigem Inhalt, der bei der direkten Bestimmung ca. 3\% Invertzucker enthielt, nach der Behandlung nach Salzsäure auch nicht mehr.

Hund Wastl: In einem entsprechenden Versuche mit $50 \mathrm{~g}$ Zucker in Substanz kam am unteren Darmende überhaupt nichts zum Vorscheine, die aus. der Duodenalkanüle kommende Flüssigkeit, von der eine Probe entnommen wurde, enthielt 38\% Rohrzucker. Reduzierender Zucker war nicht vorhanden.

Die Versuche zeigen den großen Unterschied, der besteht, je nachdem Zuckerlösungen direkt in den Dünndarm kommen oder vorher den Magen passiert haben. Die Erklärung kann nur darin gefunden werden, daß der Zucker sich im Unterschiede von Kochsalz im Darm anders verhält, wenn Salzsäure 
dabei ist, als wenn er in neutraler Lösung in den Darm kómmt. Der Rohrzucker wird relativ langsam resorbiert. ${ }^{1}$ ) Er hält damit sein Lösungswasser fest, mit dem Lösungswasser natürlich auch die anderen darin gelösten Stoffe und so kommt es, daß bei Gegenwart von Zucker die Salzsäure des Magens nicht wie sonst im Darm rasch resorbiert wird und zu wirken aufhört. Ihre Wirksamkeit wird vielmehr durch die Gegenwart des Zuckers beträchtlich verlängert. Die Wirksamkeit der Salzsäure besteht in einer Hemmung der Motilität und gleichzeitig auch der Sekretion des Magens. Unter normalen Bedingungen werden beide gleichmäßig beeinflußt. Daher wird durch die Hinzufügung des Zuckers zu anderer Nahrung die Sekretmenge und die Acidität im Magen nicht geändert, es wird vielmehr lediglich der ganze Prozeß der Magenverdauung in die Länge gezogen.

Die Verlängerung der Magenverdauung besteht, wenn Zucker allein aufgenommen wird; so erklärt sich die lange Verweildauer im Magen in den Versuchen von Best und Cohnheim. So erklärt sich wahrscheinlich auch das Sodbrennen und die Appetitsstörung, die bei manchen Menschen auftritt, wenn sie nüchtern Zucker zu sich nehmen. Denn der langen Verweildauer entspricht nicht etwa eine reichliche

- Magensaftabsonderung. Auch die Beobachtungen von Glemm am Kleinen Magen werden so verständlich.

Die Verlängerung der Magenverdauung durch den Zucker tritt aber, wie ich gezeigt habe, auch ein, wenn der Zucker bald nach einer reichlichen Mahlzeit, also auf vollen Magen, genommen wird. Damit wird dann verständlich, weshalb sich bei den meisten Kulturvölkern die Sitte eingebürgert hat, am Ende einer Mahlzeit etwas Süßes zu essen, denn durch die Zuckerwirkung wird die Verdauung der Mahlzeit über einen längeren Zeitraum hingezogen, als es sonst der Fall sein würde. Cannon ${ }^{2}$ ) hat ja gezeigt, daß das Gefühl des Hungers S. 318.

1) O. Cohnheim, Physiol. d. Verdauung u. Ernährung, Berlin 1908,

2) W.B. Cannon and A.L. Washburn, American Journ. of Physiol., Bd. 29, S. 441, 1912.

Hoppe-Seyler's Zeitschrift f. physiol. Chemie. LXXXIV. 

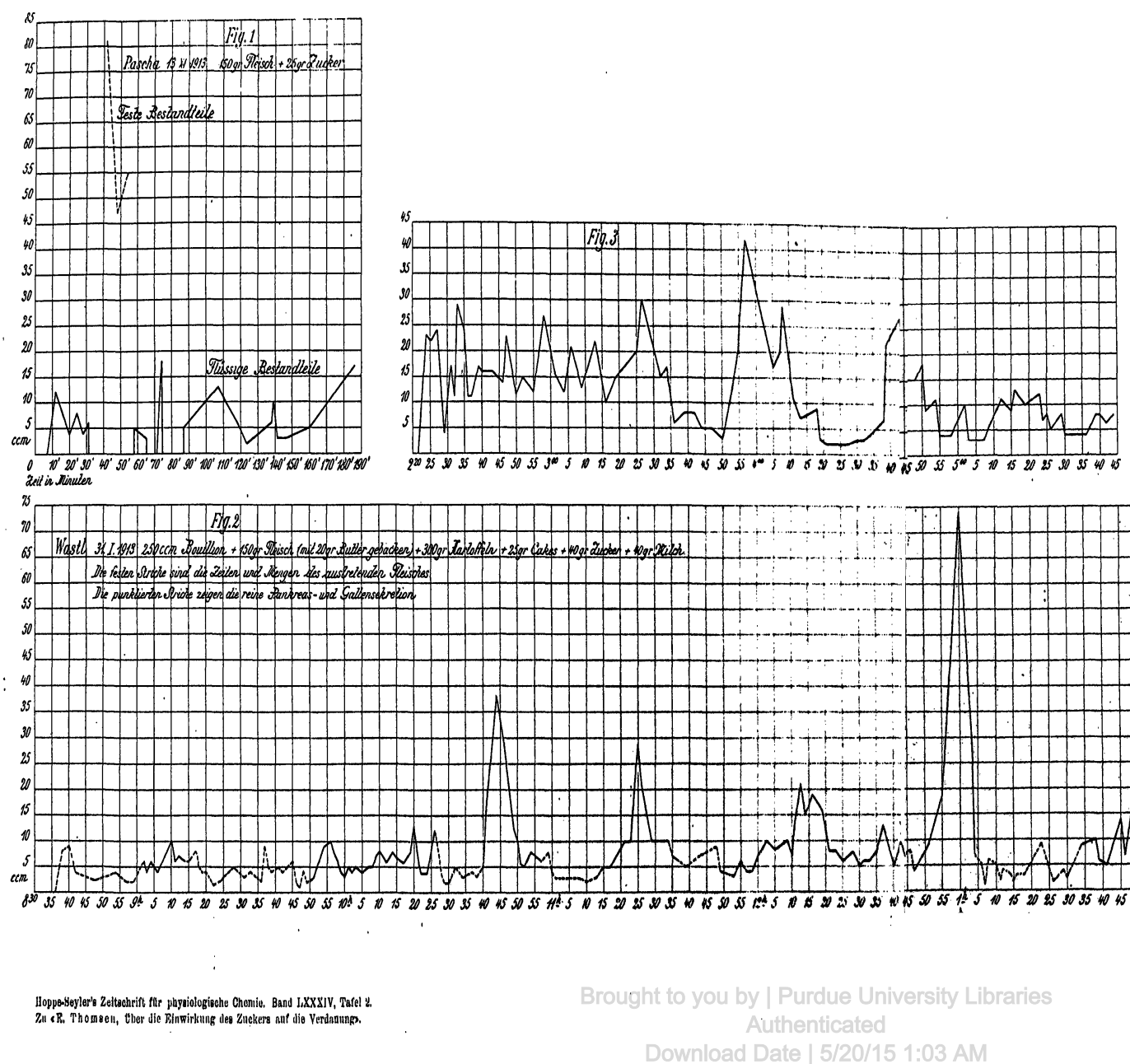
Thomsen, Üher die Einwirkung des Zuckers auf die Verdauung.

und manche andere mit ihm verbundene Magenstörungen immer auftreten, sobald der Magen eine etwas längere Zeit leer ist. Die Mahlzeiten des Menschen sind nun im allgemeinen so cingerichtet, daß der Magen fast niemals für längere Zeit leer wird. Durch die Hinzufügung von Zucker zur Mahlzeit ist es möglich, die Pause von einer Mahlzeit zur anderen zu verlängern. Das aber ist umsomehr wünschenswert, je mehr es üblich wird, die Arbeitsstätte von der Wohnung zu trennen. Ich glaube also mit diesen Untersuchungen eine physiologische Erklärung für eine weit verbreitete Sitte gegeben $z u$ haben.

\section{Zusammenfassung.}

1. Rohrzucker wirkt auf den Magen direkt nicht ein und ebensowenig auf die Sekretion von Pankreassaft und Galle. Er wirkt vom Dünndarme aus so, daß er die Magenverdauung in die Länge zieht, ohne die Sekretion zu verändern.

2. Die Verlängerung der Magenverdauung kommt so zustande, daß in die Entleerung des Magens längere Pausen eingeschaltet werden, während deren Pankreassaft und Galle sich ergießen, der Magen sich aber nicht entleert.

3. Die Wirkung des Zuckers auf den Dünndarm beruht darauf, daß er die Resorption des Speisebreies verlangsamt und damit die Wirkung der Salzsäure verlängert.

4. Rohrzucker wird selbst in sehr großen Quantitäten im Dünndarme vollständig oder fast vollständig resorbiert. 